\title{
Phenotypic plasticity and developmental noise in hybrid and parental clones of Daphnia longispina complex
}

\author{
Piotr Bernatowicz $\mathbb{E}$ ・ Piotr Dawidowicz $・$ Joanna Pijanowska
}

Received: 15 April 2021/Accepted: 23 August 2021/Published online: 8 September 2021

(C) The Author(s) 2021

\begin{abstract}
According to the "temporal hybrid superiority hypothesis", seasonal variability in environmental factors in temperate lakes gives hybrid clones within the D. longispina complex a temporary fitness advantage, thus allowing long-term, dynamic coexistence of hybrids and maternal taxa. However, the maintenance of hybrids would not require their superiority under any given set of environmental conditions if their average fitness over longer periods surpassed that of more specialized and less flexible parental clones. Phenotypic plasticity and developmental noise of several hybrid and maternal clones of Daphnia (Daphnia galeata, Daphnia hyalina, their hybrids and backcrosses) were compared in a series of laboratory experiments. Changes in depth selection and body size at first reproduction were scored in Daphnia exposed to predator (planktivorous fish) threat, to the presence of filamentous cyanobacteria
\end{abstract}

Handling editor: S.S.S. Sarma

P. Bernatowicz $(\bowtie) \cdot$ P. Dawidowicz · J. Pijanowska Department of Hydrobiology, Faculty of Biology At Biological and Chemical Research Centre, University of Warsaw, Żwirki i Wigury 101, 02-089 Warsaw, Poland e-mail: p.p.bernatowicz@uw.edu.pl

Present Address:

P. Bernatowicz

Department of Animal Physiology, Faculty of Biology, University of Warsaw, Miecznikowa 1, 02-096 Warsaw, Poland
(Cylindrospermopsis raciborskii), and to the presence of toxic compounds (PCB52 and PCB153). The hybrid clones were found to exhibit the broadest phenotypic plasticity of the studied traits in response to the different stress factors. Developmental noise in depth selection behaviour was the lowest in Daphnia galeata, the highest in Daphnia hyalina, and intermediate in hybrid and backcross clones. This diversity of reaction norms might permit the coexistence of closely related Daphnia clones in the variable and often unpredictable lake environment.

Keywords Phenotypic plasticity - Developmental noise $\cdot$ Behaviour $\cdot$ Body size $\cdot$ Hybrids $\cdot$ Daphnia longispina complex

\section{Introduction}

Cladocerans from the Daphnia longispina complex are common in European lowland lakes (Wolf and Mort 1986; Schwenk and Spaak 1997; Thielsch et al. 2017). They are keystone filter feeders which can control the biomass of lake phytoplankton and constitute an essential component of the diet of planktivorous fish (Lampert 1987; Miner et al. 2012). The Daphnia longispina group is comprised of a number of closely related species, including $D$. hyalina Leydig and D. galeata Sars, which may cross-breed and 
produce hybrids that are able to mate with each other or with non-hybrid individuals to produce backcrosses.

Under favourable conditions (during the summer), Daphnia reproduce parthenogenetically and the parental species, hybrids and backcross clones may coexist despite their almost identical morphology and diet (Wolf and Mort 1986; Spaak and Hoekstra 1995; Lynch et al. 1998). In lowland lakes, the share of hybrids in the total population of Daphnia from the $D$. longispina complex varies between 10 and $40 \%$ (Brzeziński et al. 2012), but it can temporarily exceed $80 \%$ (Spaak et al. 2004). According to the "frozen niche hypothesis" (Vrijenhoek 1978), different clones may be particularly suited to specific environmental conditions and this might explain their coexistence in spatially and/or temporally heterogeneous environments. The idea that seasonal variability in predation (Spaak and Hoekstra 1997; Spaak et al. 2000), parasite pressure (Wolińska et al. 2006), food quality (Brzeziński and Von Elert 2007) and other environmental factors gives a temporary fitness advantage to either parental species or hybrids within the D. longispina complex is a cornerstone of the "temporal hybrid superiority hypothesis" (Spaak and Hoekstra 1995), which explains the long-term dynamic coexistence of the taxa. Individual clones from natural Daphnia populations do indeed differ in predator avoidance efficiency (De Meester et al. 1995), competitive ability (Weider and Pijanowska 1993) and food quality requirements (Hairston et al. 2001; Brzeziński and Von Elert 2007; Brzeziński 2014). On the other hand, hybrid clones may dominate in both predator-free and risky habitats due to their broader plasticity when responding to variations in predation regime (Boersma et al. 1998; Bernatowicz 2006). Baker (1965) proposed the general-purpose genotype hypothesis, where a single genotype is able to produce different phenotypes across a range of environmental conditions which results in the maintenance of high fitness regardless of habitat. The "general-purpose genotype" and "frozen niche-variation" concepts are often considered as mutually exclusive, viewing clonal lineages as either generalists or specialists. Both are well supported in ecological and phylogeographic studies. Sometimes, however, generalist and specialist traits are found together in a range of asexual organisms (Vrijenhoek and Parker 2009). Relatively broad phenotypic plasticity of hybrids was indeed found upon examination of a limited number of clones (Pijanowska et al. 2007). This high phenotypic plasticity can explain the maintenance of hybrids in lakes with well-established populations of parental species. In a varying environment, the fitness of hybrids averaged over longer periods may surpass that of more specialized but less flexible parental clones, and this would not require the temporal superiority of hybrids under any given set of environmental conditions.

The broad phenotypic plasticity and developmental noise of Daphnia may be due to the very unusual genome structure. It is relatively small (200 megabases) as compared to other crustaceans but contains much more genes (at least 30,907). The high gene count is a consequence of an elevated rate of gene duplication (Colbourne et al. 2011; Tautz 2011). To date, multiplications of genes encoding, e.g., digestive enzymes (Schwarzenberger and Fink 2018) and proteins of the biological clock (Bernatowicz et al. 2016), have been found. It can be assumed that the great variety of potential gene versions is even multiplied in hybrid organisms.

The aim of this study was to test the assumption that the phenotypic plasticity (reaction norm) in depth selection behaviour and size at the first reproduction of hybrid clones from the Daphnia longispina complex exposed to various stress factors (scent of vertebrate predators, cyanobacterial filaments and PCBs) is broader than that of parental clones.

We selected one behavioural trait (the depth selection) and one life history trait (body size at first reproduction), both crucial for Daphnia individual fitness (Gliwicz 2003; Lampert 2011). We applied several environmental stress agents, among them biotic factors such as fish and cyanobacteria presence and abiotic factors, such as PCB and their various combinations. Avoiding visual predation in surface waters is a daily challenge for Daphnia, in a typical reservoir inhabited by planktivorous fish. Progressing eutrophication and climate change promote expansion of cyanobacteria that are increasing in biomass and abundance in freshwater ecosystems, often forming harmful blooms (O'Neil et al. 2012). PCBs belong to the hazardous, persistent organic pollutants (POP) of anthropogenic origin, and we selected it as a model of widespread anthropogenic factors that are commonly confronted by aquatic animals (Carlsson et al. 2018). PCBs were used extensively during the 1950-1970s, 
mostly in industrial and construction applications. Later, the production and use of PCBs were gradually restricted in many countries, and global emission decreased. However waste dumps and in-use products continue to release PCBs to the atmosphere. Together with re-emissions of PCBs accumulated in the environment, they constitute the principal sources of PCBs nowadays. Under this, recently typical for freshwaters, combination of stress agents (Birk et al. 2020), both traits that are essential for Daphnia performance (depth selection and life history traits) are known to undergo several adaptive shifts to ensure survival and reproduction.

Despite their genetic identity, individuals within the same clone do not behave in the same way, even under the same environmental conditions, nor do they have the same morphology. This phenotypic variance is called developmental noise and is explained by the microheterogeneity of the environment or by epigenetically determined variability in a population of identical individuals, as has been described in aphid populations (Babbitt 2008). Developmental noise, which results from stochasticity in molecular and cellular processes during development when genotype and environment are fixed, contributes to overall phenotypic variation (Kiskowski et al. 2019), though its role is often underestimated or neglected in studies of phenotypic variation. The contribution of developmental noise to phenotypic variation must be, however, taken into account to quantify the role of stochasticity in shaping phenotypic variation and to fully recognize the potentially adaptive role of phenotypic diversity (Draghi 2020). Mechanisms by which organisms generate adaptive functional diversity basing on such stochasticity are poorly understood. Modern thinking on phenotypic plasticity involves the concept of "mosaic physiology" exhibited by multicellular organisms. Mosaic physiology refers to assemblage of diversified phenotypes within single organisms that increase the chance of successfully coping with environmental challenges (Woods 2014). Daphnia individuals within a single clone (genotype) are known to phenotypically differ from each other due to developmental noise (Yampolsky and Scheiner 1994), but there are no data concerning this phenomenon in the Daphnia longispina group. Therefore, the second aim of this study was to measure developmental noise in depth selection behaviour and body size of parental and hybrid clones from the
Daphnia longispina complex exposed to the scent of vertebrate predators, cyanobacterial filaments and PCBs.

\section{Methods}

Identification of clones

Eleven clones from the Daphnia longispina group, originating from Lake Constance in Germany and Lakes Ros and Swiecajty in Poland, were used in this experiment. Founder individuals were captured in open water or hatched from resting eggs (ephippia) isolated from bottom sediments.

The clones were identified by electrophoretic analysis of two allozymes: AAT (aspartate transaminase, E.C. 2.6.1.1.) and AO (aldehyde oxidase, E.C. 1.2.3.1.) (Hebert and Beaton 1993). AAT and AO are diagnostic markers widely used to identify species within the Daphnia longispina complex (Wolf and Mort 1986; Gießler 1997). According to Nason and Ellstrand (Nason and Ellstrand 1993), these two markers can be used to distinguish between the parental species, their hybrids (D. galeata $\times D$. hyalina) and backcross clones (D. galeata $3 / 4$ and $D$. hyalina $3 / 4$, respectively).

\section{Experimental design}

Daphnia were maintained as stock cultures $\left(18{ }^{\circ} \mathrm{C}\right.$, 12L:12D), at an average density of $10 \mathrm{ind} \cdot \mathrm{L}^{-1}$, in aged and aerated lake water that had been filtered through a ceramic column filter (pore diameter $0.3 \mu \mathrm{m}$ ). They were fed the green algae Acutodesmus obliquus from a laboratory batch culture (in Z4 medium; Zehnder and Gorham 1960) at a non-limiting concentration of approximately $2 \mathrm{mg} \mathrm{C}_{\mathrm{org}} \cdot \mathrm{L}^{-1}$. Experimental animals originated from the third broods of mothers (originating from a single female) raised for three generations in a temperature-controlled room $\left(20^{\circ} \mathrm{C}, 16 \mathrm{~L}: 8 \mathrm{D}\right)$. The newborns (aged 0-8 h) were transferred to a plankton organ (Dawidowicz and Loose 1992), composed of glass flow-through tubes (length $60 \mathrm{~cm}$, diameter $1.5 \mathrm{~cm}$, closed at the bottom by fine net of mesh size $50 \mu \mathrm{m}$ ), arranged vertically in a transparent aquarium. Cooling of the bottom strata and heating of the surface waters of the aquarium produced a temperature gradient in the tubes that mimicked the 
summer thermal stratification in a dimictic lake (Fig. 1).

The flow of medium through the glass tubes was driven by a multi-channel peristaltic pump (Ismatec). The flow rate was set at $31.25 \mathrm{~mL} \cdot \mathrm{h}^{-1}$, which resulted in the complete exchange of medium containing the appropriate stress agents twice a day, in each tube. The plankton organ was illuminated from above by halogen bulbs shining through a frosted glass diffuser, with a photoperiod equivalent to summer light conditions (16L:8D). There were no other light sources in the room.

Cohorts of 0-8-h-old neonates were divided between the tubes, at 7-9 animals per tube. The position of all animals was recorded with $1 \mathrm{~cm}$ accuracy twice a day ( 4 and $8 \mathrm{~h}$ after the laboratory sunrise). Experiments were terminated when the Daphnia in all treatments released the 1 st clutch of eggs into their brood chambers. Individual clones were tested separately.

After behavioural observations, all Daphnia were removed from the plankton organ and photographed using a digital camera attached to a microscope. The body size of each individual (20 individuals from each variant) was measured from this image using Multiscan ${ }^{\circledR}$ software as the distance between the lower edge of the eye and the base of the tail spine.

In all, eleven clones of Daphnia were examined: 2 D. galeata, 2 D. galeata $3 / 4,2$ hybrids, 2 D. hyalina $3 / 4$ and 3 D. hyalina. The Daphnia were subjected to 12 treatments using growth medium containing different

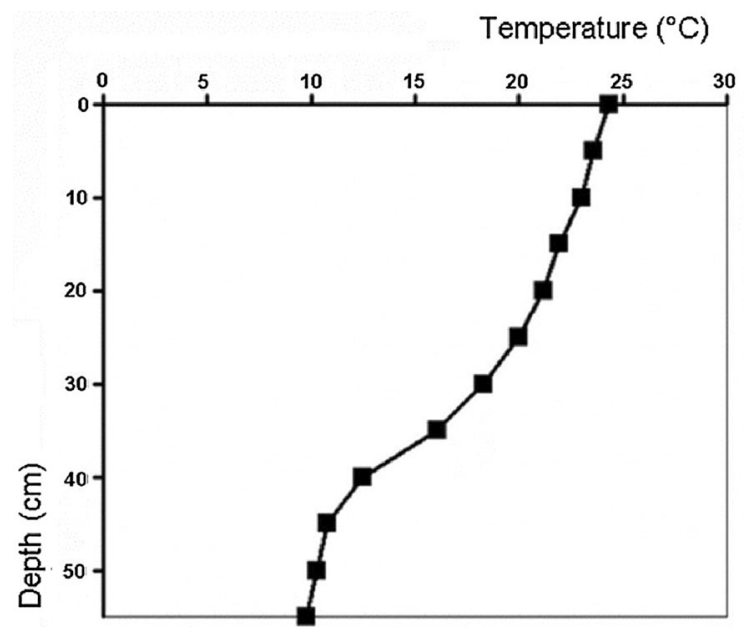

Fig. 1 Temperature gradient in the plankton organ biotic and abiotic stress factors: (1) PCB 153; (2) PCB 52; (3) fish kairomone; (4) cyanobacteria Cylindrospermopsis raciborskii; (5) fish kairomone and cyanobacteria; (6) fish kairomone and PCB 153; (7) fish kairomone and PCB 52; (8) cyanobacteria and PCB 153; (9) cyanobacteria and PCB 52; (10) fish kairomone and cyanobacteria and PCB 153; (11) fish kairomone and cyanobacteria and PCB 52; and (12) control. Two replicates of each treatment and Daphnia clone were performed.

The fish kairomone medium was obtained by placing a single rudd (Scardinius erytrophtalmus, $8 \mathrm{~cm}$ ) fed with Daphnia in $5 \mathrm{~L}$ of conditioned lake water for $24 \mathrm{~h}$. The non-toxic strain of $C$. raciborskii was batch-cultured in Z4 medium and filaments were harvested upon reaching stationary growth phase. Under such conditions, this cyanobacterium does not produce toxins and its negative impact on Daphnia can be attributed to mechanical interference of the filaments with food collection and/or their low nutritional value (Bednarska et al. 2014). For the cyanobacteria treatment, $C$. raciborskii was added to the medium at a concentration of $0.5 \mathrm{mg} \mathrm{C}$ org $\cdot \mathrm{L}^{-1}$, which is equivalent to $\sim$ 3200-3400 filaments $\mathrm{mL}^{-1}$. Similar filament densities have been widely used in assays testing the impact of filamentous cyanobacteria on filter feeding zooplankton (Abrusán 2004; Bednarska and Dawidowicz 2007). In the treatments with PCBs, congeners 153 and 52 were diluted in acetone and added to the medium at a concentration of $10 \mu \mathrm{g} \mathrm{L}^{-1}$. Acetone at such low concentrations has no effect on Daphnia and is lost from the solution by evaporation (Ulmann 1972; Ferrando et al. 1996; Sánchez et al. 2000).

Statistical analyses

We applied the analysis of regression to check if there is a relationship between the degree of hybridization of Daphnia clones and measures of phenotypic plasticity and development noise.

The phenotypic plasticity (PP) was calculated for each Daphnia clone according to the formula proposed by Valladares (Valladares et al. 2000). The developmental noise (DN) was calculated using the same formula, modified to take into account variation within the treatments, not between them. Values of zero indicate no plasticity or noise, whereas values of 1 indicate full plasticity or noise (Eqs. 1-4). 
$\mathrm{PP}_{\text {depthselection }}=\left(X_{\max }-X_{\min }\right) / h$

$\mathrm{PP}_{\text {bodysize }}=\left(X_{\max }-X_{\min }\right) / X_{\max }$

where: PP-phenotypic plasticity. $X_{\max }$-maximum mean of Daphnia body size or selected depth among all treatments. $X_{\min }$-minimum mean of Daphnia body size or selected depth by among all treatments. $H$-height of water column.

The maximum and the minimum means were the average values, the highest and the lowest among means calculated for each treatment within a single clone.

$\mathrm{DN}_{\text {depth selection }}=\frac{\sum_{i=1}^{n}\left(X_{\max i}-X_{\min i}\right) / h}{n}$

$\mathrm{DN}_{\text {body size }}=\frac{\sum_{i=1}^{n}\left(X_{\max i}-X_{\min i}\right) / h}{n}$

where: DN-developmental noise. $N$-number of treatments in whole experiment. $x_{\max }-$ maximum selected depth or body size of a single Daphnia individual in treatment $i . x_{\min } i$-minimum selected depth or body size of a single Daphnia individual in treatment $i$. $h$-height of water column. $x_{\max }-\max -$ imum depth or body size of Daphnia, noticed across all treatments.

Phenotypic plasticity and developmental noise were calculated separately for depth selection behaviour and body length for each of the 11 clones in two replications. To estimate the statistical significance of differences in the values of PP and DN for "pure" $D$. hyalina and D. galeata, and hybrids, we conducted analysis of regression using values of phenotypic (PP) plasticity, developmental noise (DN), both in two replications, and estimated contribution of $D$. hyalina genes to the genome. It was assumed that estimated contribution equals 0 for "pure" $D$. galeata, 0.25 for D. galeata $3 / 4$ clones, 0.5 for hybrids, 0.75 for $D$. hyalina $3 / 4$ clones and 1 for "pure" $D$. hyalina. This simplification permitted the arrangement of the examined clones for further correlation procedures.

Regression analyses were calculated using Statistica v.13.3 software.

\section{Results}

One of the parental species, D. galeata, stayed close to the water surface and had the narrowest range of selected depths, while the other, D. hyalina, preferred deeper in the colder water layers and had a broader range of selected depths. Hybrid clones exhibited the broadest range of average selected depths. Depending on the treatment, individuals from this group were detected close to surface or in the cold water layers near the bottom (Fig. 2).

D. galeata individuals were larger than those of $D$. hyalina, whereas hybrid and backcross clones had intermediate body sizes. As with depth selection range, the average body size range was smaller in parental clones than in hybrids and backcrosses (Fig. 2).

The highest values of behavioural phenotypic plasticity were observed in hybrid and backcross clones, while D. galeata clones exhibited the lowest values (Fig. 3). There was no correlation between the estimated contribution of $D$. hyalina genes to the genome and phenotypic plasticity in depth selection (Fig. 4).

The highest values of phenotypic plasticity in body size were observed in hybrid and backcross clones, while the parental clones exhibited the lowest values (Fig. 3). In this case, there was a significant polynomial correlation between the estimated contribution of $D$. hyalina genes to the genome and phenotypic plasticity in body size (Fig. 4).

Behavioural developmental noise attained high values in D. hyalina and the hybrid clones, while the lowest values were observed in D. galeata (Fig. 3). There was a linear correlation between the estimated contribution of $D$. hyalina genes to the genome (Fig. 5) and behavioural developmental noise. Values of developmental noise in body size were similar in all tested clones (Fig. 3) and did not correlate with the estimated contribution of $D$. hyalina genes to the genome (Fig. 5).

We did not find any significant effect of the origin of animals (ephippia vs. open water, different lakes) on phenotypic plasticity or developmental noise. 

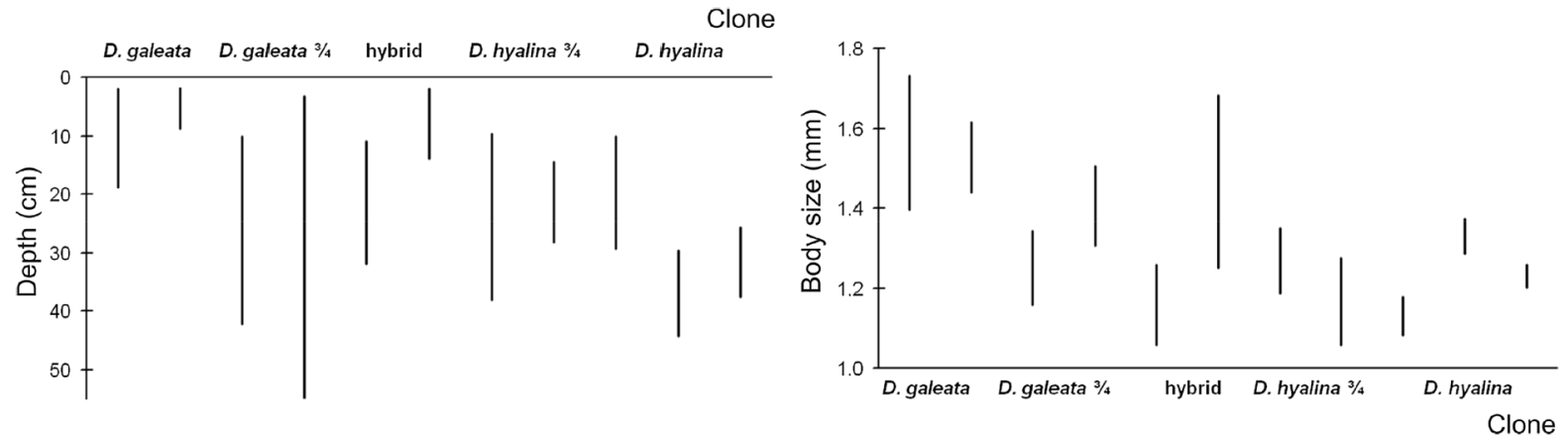

Fig. 2 Breadth of reaction based on depth selection (left) and body size (right) in eleven Daphnia longispina clones exposed to various experimental treatments

Depth selection
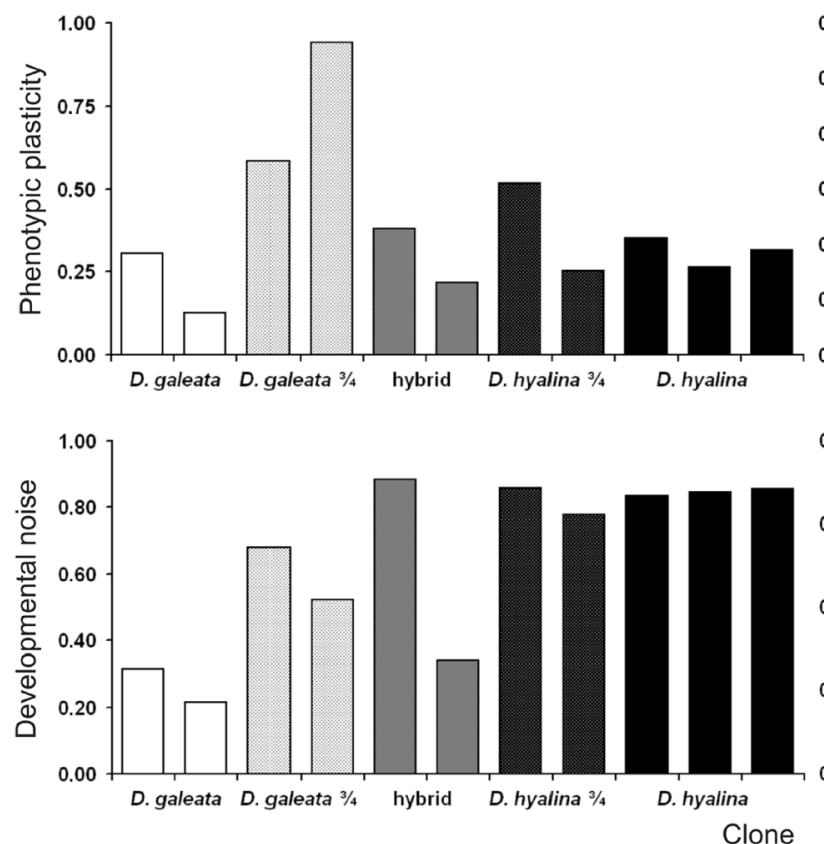

Body size
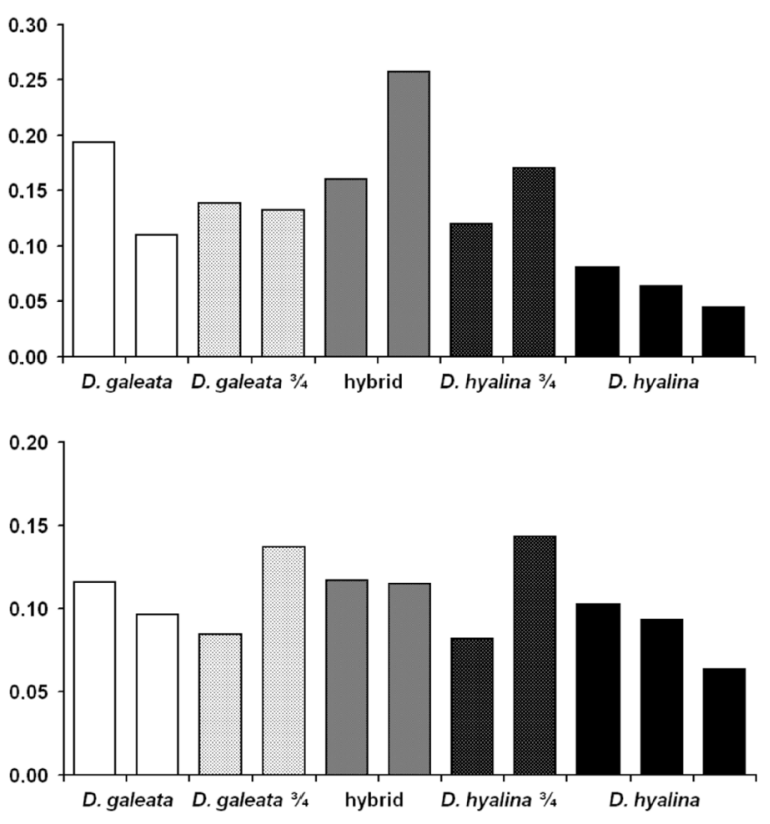

Fig. 3 Phenotypic plasticity and developmental noise (mean) based on depth selection behaviour and body size in eleven Daphnia longispina clones

\section{Discussion}

Environmental conditions in lakes vary considerably throughout the season and, also, change within $24 \mathrm{~h}$ cycles, especially for migrating animals that explore the broad range of both, abiotic and biotic variability when descending to the bottom at the dawn and ascending to the surface after the dusk. For example, the level of predator pressure or the density of harmful cyanobacteria may change in sudden and unexpected ways (Lampert 1987; Janse et al. 2005). Since
Daphnia are unable to predict small scale, local changes in abiotic factors such as light intensity, temperature or the presence of toxic compounds, the ability to modify phenotype enhances the relative fitness of a clone in a changing environment when compared to clones with a constitutive phenotype (Moran 1992; Pijanowska 1993). Here, we show that within the D. longispina group, differences in phenotypic plasticity and developmental noise exist between parental species and hybrids, which may be the basis for the reported temporal dominance and permanent 

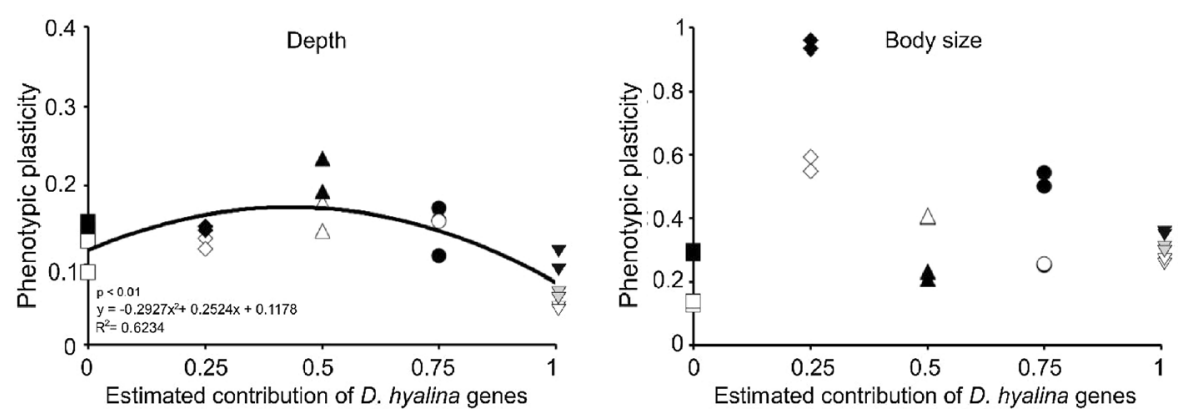

Fig. 4 Relation between estimated contribution of $D$. hyalina genes in the genome and phenotypic plasticity based on depth selection (left) and body size (right). (Symbols represent different clones)
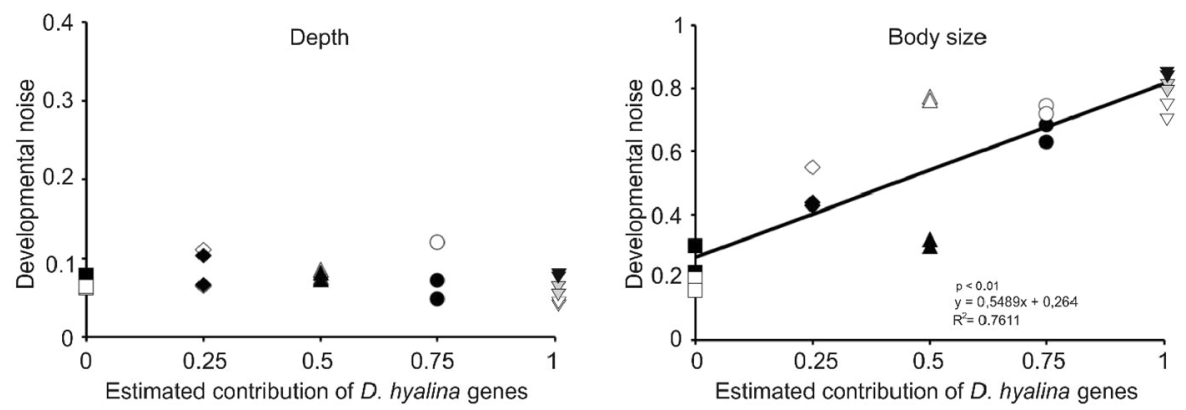

Fig. 5 Relation between estimated contribution of D. hyalina genes to the genome and developmental noise based on depth selection (left) and body size (right). (Symbols represent different clones)

coexistence of the taxa. According to Spaak and Hoekstra (1997), the spatio-temporal heterogeneity of pelagic biota may explain the coexistence of Daphnia hybrids with parental species, as it can reduce the time available for a temporarily best-fit clone to arise and out-compete the others.

The hybrid Daphnia within D. longispina species complex were found to have greater phenotypic plasticity (broader reaction norm) than the parental species, which may be related to their overall higher heterozygosity compared to the parent species (Griebel et al. 2016). This may indicate that hybrids are better adapted to spatio-temporal environmental heterogeneity and thus their coexistence with (or even dominance over) parental species may result from their ability to cope better with a varying environment. Genotypes that perform best in one environment usually perform less well in another one, their performance being not an adaptation to environmental variation. A response to environmental variation is adaptive if relatively higher fitness can be maintained in the changing environment (Thomson 1991). For example, sexual reproduction in Daphnia populations, thus also hybrid formation, occurs mainly late in the season, so that the majority of dormant (ephippial) eggs are produced by parents from clones that perform best in the fall. Consequently, ex-ephippial Daphnia that hatch next spring are burdened with adaptations of their parents not necessarily best suited to the current environmental conditions. If this is the case, the relatively high phenotypic plasticity of hybrids gives them an early season advantage over less flexible individuals from "pure" parental species lineages, something that may result in numerical dominance of hybrids within the $D$. longispina complex in spring (e.g. Yin et al. 2014). As suggested by Boriss and Gabriel (1998), phenotypic plasticity in depth selection behaviour enables hybrid populations to successfully invade an environment even though the invaders have less efficient physiology and morphology than the established parental species populations. This would also explain the frequently observed dominance of hybrids in such variable environments.

The broader phenotypic plasticity of hybrids is a phenomenon that has been described in many different species, and may be explained by the inheritance of a 
set of genes from both parents. Depending on the environmental conditions encountered, genes from either of the parental sets may be activated (Scheiner 1993), or interaction between the two sets might occur (Smith 1990) to produce a novel phenotype.

Developmental noise in behavioural traits has not previously been described for Daphnia longispina clones. In most statistical analyses of Daphnia behaviour or life histories, the phenomenon of developmental noise is ignored, and its effect is treated as the built-in residual error. To estimate differences in developmental noise between examined groups of organisms (e.g. Daphnia clones), the reduction in other variability-inducing factors is needed. In this study, genetic variability was eliminated by using genetically identical, clonal individuals. Significantly more developmental noise was detected in D. hyalina than in D. galeata. An increase in phenotypic variance, expressed as the developmental noise, results in idiosyncratic and unpredictable responses of individuals to biotic environment. This unpredictability of the reactions of individuals may reflect the possible differences in their personalities. However, the personalities among clonal planktonic crustaceans have not been studied at all so far. The production of phenotypically diverse offspring in $D$. hyalina may be interpreted as an expression of a bethedging (risk-spreading) strategy (Slatkin 1974) employed by this species when it faces variable environments. Moreover, it may reduce the likelihood of evolution of manipulative strategies in interacting partners (Fordyce 2006).

Acknowledgements We are thankful to the Editors of this volume for kindly inviting us to honour the memory of our unforgettable friend, Professor Ramesh Gulati. We thank Prof. Małgorzata Kloc for critical reading of this manuscript and Dr. Tomasz Brzeziński for his help in experimental work and for his comments to the text. We are also grateful to the Anonymous Reviewers whose comments helped to improve and modernize our contribution. This study was supported by grants from the State Committee for Scientific Research (KBN) in Poland (nos. 2PO4F 06727 and 4136/B/PO1/2007/33).

\section{Declarations}

Conflict of interest The authors declare that they have no conflict of interest and all institutional and national guidelines for the care and use of laboratory animals were followed.
Data availability The data that support the findings of this study are not openly available and are available from the corresponding author upon reasonable request.

Open Access This article is licensed under a Creative Commons Attribution 4.0 International License, which permits use, sharing, adaptation, distribution and reproduction in any medium or format, as long as you give appropriate credit to the original author(s) and the source, provide a link to the Creative Commons licence, and indicate if changes were made. The images or other third party material in this article are included in the article's Creative Commons licence, unless indicated otherwise in a credit line to the material. If material is not included in the article's Creative Commons licence and your intended use is not permitted by statutory regulation or exceeds the permitted use, you will need to obtain permission directly from the copyright holder. To view a copy of this licence, visit http://creativecommons.org/licenses/by/4.0/.

\section{References}

Abrusán G (2004) Filamentous cyanobacteria, temperature and Daphnia growth: the role of fluid mechanics. Oecologia 141:395-401

Babbitt DA (2008) How accurate is the phenotype?-An analysis of developmental noise in a cotton aphid clone. BMC Dev Biol. https://doi.org/10.1186/1471-213X-8-19

Baker HG (1965) Characteristics and modes of origin of weeds. In: Baker HG, Stebbins GL (eds) Genetics of colonizing species. Academic Press, New York, pp 147-172

Bednarska A, Dawidowicz P (2007) Change in filter-screen morphology and depth selection: uncoupled responses of Daphnia to the presence of filamentous cyanobacteria. Limnol Oceanogr 52:2358-2363

Bednarska A, Pietrzak B, Pijanowska J (2014) Effect of poor manageability and low nutritional value of cyanobacteria on Daphnia magna life history performance. J Plankton Res 36:838-847

Bernatowicz P (2006) Phenotypic plasticity and developmental noise in Daphnia longispina group. Ver Internat Limnol 29:1535-1538

Bernatowicz P, Kotwica-Rolinska J, Joachimiak E, Sikora A, Polanska MA, Pijanowska J, Bębas P (2016) Temporal expression of the clock genes in the water flea Daphnia pulex (Crustacea: Cladocera). J Exp Zool A 325:233-254

Birk S, Chapman D, Carvalho L et al (2020) Impacts of multiple stressors on freshwater biota across spatial scales and ecosystems. Nat Ecol Evol 4:1060-1068

Boersma M, Spaak P, De Meester L (1998) Predator-mediated plasticity in morphology, life history, and behavior of Daphnia: the uncoupling of responses. Am Nat 152:236-248

Boriss H, Gabriel W (1998) Vertical migration in Daphnia: the role of phenotypic plasticity in the migration pattern for competing clones or species. Oikos 83:129-138

Brzeziński T, Von Elert E (2007) Biochemical food quality effects on a Daphnia hybrid complex. Limnol Oceanogr 52:2350-2357 
Brzeziński T, Fronk J, Trzcińska-Danielewicz J, Dawidowicz P (2012) Interspecific hybridization in sympatric species of Daphnia inhabiting lakes in northeastern Poland. Ocean Hydrobiol Stud 41:1-6

Carlsson P, Breivik K, Brorström-Lundén E et al (2018) Polychlorinated biphenyls (PCBs) as sentinels for the elucidation of Arctic environmental change processes: a comprehensive review combined with ArcRisk project results. Environ Sci Pollut Res Internat 25:22499-22528

Colbourne JK, Pfrender ME, Gilbert D et al (2011) The ecoresponsive genome of "Daphnia pulex". Science 331(6017):555-561

Dawidowicz P, Loose CJ (1992) Metabolic costs during predator-induced diel vertical migration in Daphnia. Limnol Oceanogr 37:665-669

De Meester L, Weider LJ, Tollrian R (1995) Alternative antipredator defences and genetic polymorphism in a pelagic predator-prey system. Nature 378:483-485

Draghi J (2020) Developmental noise and ecological opportunity across space can release constraints on the evolution of plasticity. Evol Develop 22:35-46

Ferrando MD, Sancho E, Andreau-Moliner E (1996) Chronic toxicity of fenitrothion to an algae (Nannochloris oculata), a Rotifer (Brachionus calyciflorus), and the Cladoceran (Daphnia magna). Ecotox Environ Saf 35:112-120

Fordyce JA (2006) The evolutionary consequences of ecological interactions mediated through phenotypic plasticity. J Exp Biol 209:2377-2383

Gießler S (1997) Gene flow in the Daphnia longispina hybrid complex (Crustacea, Cladocera) inhabiting large lakes. Heredity 79:231-242

Gliwicz ZM (2003) Between hazards of starvation and risks of predation: the ecology of offshore animals. International Ecology Institute, Oldendorf/Luhe

Griebel J, Gießler S, Yin M, Wolinska J (2016) Parental and hybrid Daphnia from the D. longispina complex: longterm dynamics in genetic structure and significance of overwintering modes. J Evol Biol 29:810-823

Hairston NG Jr, Holtmeier CL, Lampert W, Weider LJ, Post DM, Fischer JM (2001) Natural selection for grazer resistance to toxic cyanobacteria: evolution of phenotypic plasticity? Evolution 55:2203-2214

Hebert PDN, Beaton MJ (1993) Methodologies for allozyme analysis using cellulose acetate electrophoresis, a handbook. Helena Laboratories, Beaumont

Janse I, Kardinaal WE, Agterveld MK, Meima M, Visser PM, Zwart G (2005) Contrasting microcystin production and cyanobacterial population dynamics in two Planktothrixdominated freshwater lakes. Environ Microbiol 7:1514-1524

Kiskowski M, Glimm T, Moreno N, Gamble T, Chiari Y (2019) Isolating and quantifying the role of developmental noise in generating phenotypic variation. PLoS Comput Biol 15:e1006943

Lampert W (1987) Feeding and nutrition in Daphnia. In: Peters RH, De Bernardi R (Eds.) Daphnia. Consiglio Nazionale Delle Ricerche Istituto Italiano di Idrobiologia, Verbania Palanza, Italy.

Lampert W (2011) Daphnia: development of model organism in ecology and evolution. International Ecology Institute, Oldendorf/Luhe
Lynch M, Latta L, Hicks J, Giorgianni M (1998) Mutation, selection, and the maintenance of life-history variation in a natural population. Evolution 52:727-733

Miner BE, De Meester L, Pfrender ME, Lampert W, Hairston NG Jr (2012) Linking genes to communities and ecosystems: Daphnia as an ecogenomic model. Proc R Soc B 279:1873-1882

Moran NA (1992) The evolutionary maintenance of alternative phenotypes. Am Nat 128:971-978

Nason JD, Ellstrand NC (1993) Estimating the frequencies of genetically distinct classes of individuals in hybridized populations. J Hered 84:1-12

O’Neil JM, Davis TW, Burford MA, Gobler CJ (2012) The rise of harmful cyanobacteria blooms: the potential roles of eutrophication and climate change. Harmful Algae 14:313-334

Pijanowska J (1993) Diel vertical migration in zooplankton: fixed or inducible behavior? Arch Hydrobiol 39:89-97

Pijanowska J, Bernatowicz P, Fronk J (2007) Phenotypic plasticity within Daphnia longispina complex: differences between parental and hybrid clones. Pol J Ecol 55:761-769

Sánchez M, Ferrando MD, Sancho E, Andreu E (2000) Physiological perturbations in several generations of Daphnia magna Straus exposed to diazinon. Ecotox Environ Saf 46:87-94

Scheiner SM (1993) Genetics and evolution of phenotypic plasticity. An Rev Ecol Systemat 24:35-68

Schwarzenberger A, Fink P (2018) Gene expression and activity of digestive enzymes of Daphnia pulex in response to food quality differences. Comp Biochem Physiol B 218:23-29

Schwenk K, Spaak P (1997) Ecology and genetics of interspecific hybridization in Daphnia. In: Streit B, Stadler T, Lively CM (eds) Evolutionary ecology of freshwater animals. Birkhäuser Basel, Switzerland

Slatkin M (1974) Hedging ones evolutionary bets. Nature 250:704-705

Smith M (1990) Signal perception, differential expression within multigene families and the molecular basis of phenotypic plasticity. Plant Cell Environ 13:585-594

Spaak P, Boersma M (2000) Predator-induced life-history changes and the coexistence of five taxa in a Daphnia species complex. Oikos 89:164-174

Spaak P, Hoekstra JR (1995) Life history variation and the coexistence of a Daphnia hybrid with its parental species. Ecology 76:553-565

Spaak P, Hoekstra JR (1997) Fish predation on Daphnia hybrid species complex: a factor explaining species coexistence? Limnol Oceanogr 42:753-762

Spaak P, Denk A, Boersma M, Weider LJ (2004) Spatial and temporal patterns of sexual reproduction in a hybrid Daphnia species complex. J Plankton Res 26:625-635

Tautz D (2011) Not just another genome. BMC Evol Biol 9:8

Thielsch A, Knell A, Mohammadyari A, Petrusek A, Schwenk K (2017) Divergent clades or cryptic species? Mito-nuclear discordance in a Daphnia species complex. BMC Evol Biol 17:1-9

Thomson JD (1991) Phenotypic plasticity as a component of evolutionary change. TREE 6:246-249

Ulmann E (1972) Lindane: Monograph of an insecticide. Schillinger, Freiburg 
Valladares F, Wright SJ, Lasso E, Kitajima K, Pearcy RW (2000) Plastic phenotypic response to light of 16 congeneric shrubs from Panamanian rainforest. Ecology 81:1925-1936

Vrijenhoek RC (1978) Coexistence of clones in a heterogeneous environment. Science 199:549-552

Vrijenhoek RC, Parker ED (2009) Geographical parthenogenesis: general purpose genotypes and frozen niche variation. In: Schön I, Martens K, Dijk P (eds) Lost sex. Springer, Dordrecht

Weider LJ, Pijanowska J (1993) Plasticity of Daphnia life histories in response to chemical cues from predators. Oicos 67:385-895

Wolf HG, Mort MA (1986) Inter-specific hybridization underlies phenotypic variability in Daphnia populations. Oecologia 68:507-511

Wolińska J, Bittner K, Ebert D, Spaak P (2006) The coexistence of hybrid and parental Daphnia: the role of parasites. Proc Biol Sci 273:1977-1983
Woods HA (2014) Mosaic physiology from developmental noise: within-organism physiological diversity as an alternative to phenotypic plasticity and phenotypic flexibility. J Exp Biol 217:35-45

Yampolsky LY, Scheiner SM (1994) Developmental noise, phenotypic plasticity, and allozyme heterozygosity in Daphnia. Evolution 48:1715-1723

Yin M, Gießler S, Griebel J, Wolinska J (2014) Hybridizing Daphnia communities from ten neighbouring lakes: spatiotemporal dynamics, local processes, gene flow and invasiveness. BMC Ecol Evol 14:80

Zehnder AA, Gorham PR (1960) Factors influencing the growth of Microcystis aeruginosa Kütz emend Elenk. Can J Microbiol 6:645-660

Publisher's Note Springer Nature remains neutral with regard to jurisdictional claims in published maps and institutional affiliations. 